\title{
DIGITAL TECHNOLOGY Extraordinary virtual multidisciplinary team meetings - a novel forum for the coordinated care of patients with complex conditions within a secondary care setting
}

\author{
Authors: Stephen ] Aston, ${ }^{\mathrm{A}}$ Sophie Reade, ${ }^{\mathrm{B}}$ Barbara Petersen, ${ }^{\mathrm{C}}$ Charlotte Ward, ${ }^{\mathrm{D}}$ Anthony Duffy ${ }^{\mathrm{E}}$ and Emmanuel \\ Nsutebu ${ }^{F}$
}

\begin{abstract}
Multidisciplinary team (MDT) meetings are increasingly regarded as best practice for the successful management of chronic disease. However, for patients with undiagnosed illnesses, multiple interacting comorbidities or other complex needs that fall outside the remit of disease-specific MDTs or the scope of expertise of individual clinicians, there is often no suitable forum at which to discuss their care to develop a coordinated plan for management. We developed and piloted a new forum for interspecialty discussion and collaboration, an extraordinary virtual MDT, to enable clinicians to arrange an urgent meeting of all involved parties in response to challenging clinical scenarios. Here, we share our experience of implementing this innovation and suggest how this novel forum for coordinated care could be further developed to improve the integration, timeliness and quality of healthcare delivery for patients with complex needs.
\end{abstract}

KEYWORDS: Virtual multidisciplinary team, videoconferencing

\section{Introduction}

Multidisciplinary team (MDT) meetings have become commonplace in modern healthcare. ${ }^{1,2}$ However, for many patients with undiagnosed illnesses or complex needs, the current system of disease-specific MDTs is inadequate and there is often no alternative interspecialty forum at which to discuss their

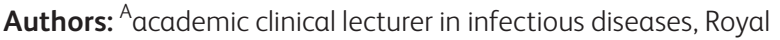
Liverpool and Broadgreen University Hospitals NHS Trust, and Institute of Infection and Global Health, University of Liverpool,

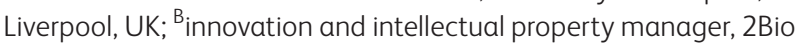
Ltd, Liverpool, UK; ${ }^{C}$ medical secretary and multidisciplinary team meeting coordinator, Royal Liverpool and Broadgreen University

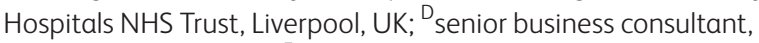
2Bio Ltd, Liverpool, UK; Esenior lean practitioner, Royal Liverpool and Broadgreen University Hospitals NHS Trust, Liverpool, UK; ${ }^{F}$ consultant infectious diseases physician and AQuA leadership for improvement fellow, Royal Liverpool and Broadgreen University Hospitals NHS Trust, Liverpool, UK care. We developed and piloted a new concept of extraordinary virtual MDT meetings for patients with complex conditions within a secondary care setting. Here, we share our experience and discuss how this novel forum for coordinated care could be further developed to improve the quality of healthcare delivery.

The increasing challenge of delivering integrated care

MDT meetings were introduced into UK practice for cancer care during the 1990s to ensure the equality of access to standardised, high-quality care and were shortly after adopted as a mandatory component of cancer case management. ${ }^{3-5}$ Patients managed via MDTs report improved satisfaction, receive more timely treatment and, for some tumour types, have improved survival. ${ }^{6-9}$ Over the past decade, MDTs in other specialties have proliferated such that most hospitals or regions have regular MDT meetings for various conditions (eg heart failure, stroke, and tuberculosis). The concept of MDT working is now widely accepted and considered good practice for the successful management of chronic disease. ${ }^{1,3}$

MDT meetings typically involve a predefined membership of clinicians meeting regularly, usually in face-to-face meetings, at a recurring fixed time to discuss patients with a specific condition. ${ }^{10}$ However, for some patients with rare or complex conditions, multiple interacting comorbidities or those without a clear diagnosis, there is often no appropriate routine MDT in which to discuss their care. These patients with complex needs, who stand to benefit from the collaborative approach of a MDT format, are often instead subject to multiple sequential reviews from various specialist teams and are at risk of experiencing delayed and fragmented care. ${ }^{11}$ In Box 1 , we describe our experience of a patient whose care was adversely affected by the absence of a suitable forum to bring together the numerous clinicians involved in their care. The parallel trends of increased life expectancy with the attendant risk of accumulating multiple chronic illnesses and the drive towards centralisation of healthcare services ${ }^{12}$ is likely to mean that a growing number of patients will have illnesses that fall outside the scope of expertise of any individual clinician or the remit of disease-specific MDTs. Without effective mechanisms for interspecialty working, such patients are at risk of fragmented care. ${ }^{11,13}$ We believe that these patients with complex conditions 
Box 1. Case study: delayed diagnosis and the need for an Ex-vMDT

An adult patient was admitted to our hospital with fever, breathlessness, weight loss, enlarged lymph nodes and abdominal swelling. Computed tomography (CT) scans showed ascites, extensive intra-abdominal nodes and enlarged hilar and peripheral nodes. Initial investigations for tuberculosis (TB) and lymphoma were performed. The case was discussed at the weekly TB multidisciplinary team (MDT) meeting and empirical TB treatment was started. Unfortunately, the patient did not improve, and TB treatment was stopped. Multiple individual specialists reviewed the patient and gave their clinical opinion about investigation and management, including a gastroenterologist, respiratory physicians, haematologist, rheumatologist, infectious diseases physicians and multiple surgeons. Attempts to obtain a diagnosis by analysing ascitic fluid and peripheral lymph node biopsy were unsuccessful. Laparoscopic biopsy of intra-abdominal lymph nodes was felt to be too risky and, hence, was avoided by the surgical team. When a decision was made to perform a surgical biopsy, the patient was too unwell and, unfortunately, died after being in hospital for 6 weeks. A post-mortem was carried out and confirmed a diagnosis of lymphoma, which was potentially treatable.

require some form of MDT that can convene quickly to develop coordinated management plans.

\section{An extraordinary virtual MDT for complex conditions}

We developed the concept of an extraordinary MDT for patients with complex conditions in which a lead clinician responsible for a patient's care could request a promptly arranged meeting of all involved parties in response to a challenging clinical scenario (eg diagnostic dilemma or need for a coordinated treatment plan). We envisaged that the discussions of complex patients would often involve clinicians from various specialties spread across different sites, such that a face-to-face meeting would be difficult to arrange at short notice. Therefore, we developed, in parallel, the technological and logistical mechanisms to enable attendees to participate remotely. Although virtual MDTs have been used in community settings, to our knowledge, this combination of an ad hoc multispecialty forum for coordinated case management facilitated by a virtual platform has not been described previously in a secondary care setting.

From June 2016 to June 2017, we conducted a pilot exercise to assess both the feasibility and utility of a system for extraordinary virtual MDTs (Ex-vMDT). The project was supported by a grant from the Innovation Agency (formerly North West Coast Academic Health Science Network) to fund the purchase of video conferencing software.

During the pilot phase, we restricted the use of the Ex-vMDT system to discuss patients who were not suitable for inclusion in another established MDT and met one or more of the following criteria: lacked a confirmed diagnosis and treatment plan; required a coordinated plan for investigation, discharge planning or follow-up; or had a complex diagnostic or therapeutic dilemma likely to benefit from a multidisciplinary approach. Patients could be referred from any consultant within the trust via an online referral system (Fig 1).
Patient identified and meets eligibility criteria for 'extraordinary MDT meeting for complex conditions



Referral form sent to MDT coordinator via online request system,

specifying reason for referral, intended outcome, urgency and

specialists required

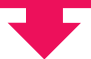

MDT coordinator contacts referring clinician for their availability within the specified timeframe (ie 24 hours, 3 days etc)

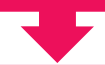

MDT coordinator contacts invitees for availability and schedules meeting

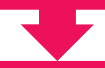

Invite sent to all participants including link for online video

conferencing software used in meeting

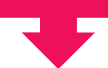

Referring clinician and MDT coordinator chair meeting, record outcome and circulate to participants

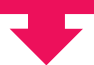

Feedback form sent to referring clinician and participants to evaluate MDT meeting and process

Fig 1. Flow chart for an extraordinary virtual multidisciplinary team (MDT) for complex conditions, illustrating processes from referral to feedback.

We used the GoToMeeting video conferencing software (LogMeIn, Inc.; Boston, USA), which provides robust end-to-end encryption and allows conferencing from multiple sites and for users to share files and screens. The outcome of the Ex-vMDT was recorded on a standard pro forma and shared with all participants, the patients' GP and, where appropriate, the patients themselves.

The pilot was evaluated by collating feedback from participants using a standardised questionnaire that asked respondents to rate their satisfaction with the process, technologies and outcome of the Ex-vMDT meeting (Fig 2). Feedback from patients discussed at the meeting was sought when appropriate.

The Ex-vMDT was instituted as a service development project; formal research ethics committee approval was not sought.

\section{Pilot study outcomes}

Over the course of the 12-month pilot, the care of five patients referred by five consultant physicians was discussed in Ex-vMDT meetings (Table 1). Three patients had unknown diagnoses and the focus of the discussion was to devise a coordinated plan for investigation. The other two patients were suspected to have primarily functional illnesses and the focus of the Ex-vMDT meetings was to agree an integrated plan of care and follow-up. Four meetings included practitioners based at different hospital sites from the requesting clinician and one involved the patient's GP.

\section{Clinician feedback}

The feedback from the nine clinicians who completed the feedback survey suggests that not only was the Ex-vMDT meeting 
Table 1. Patients discussed during the Ex-vMDT meeting system pilot study

Case

Medical inpatient with pre-existing chronic pelvic pain admitted with medically unexplained paraplegia and sensory deficits

Complex outpatient with background of chronic liver and kidney disease under review for nonresolving pulmonary mass

Acute kidney injury because of suspected microangiopathy following diarrhoeal illness and unexplained ascites requiring recurrent paracentesis

6-month inpatient stay with acute cerebellar disorder of unknown cause complicated by recurrent aspiration pneumonia and intra-abdominal sepsis

Chronic dysphagia, vomiting and malnutrition. Recurrent admissions with complications related to gastrostomy feeding and parenteral nutrition

\section{Summary of MDT}

MDT involving clinicians across three hospitals: gynaecologist, general physician and physiotherapist; psychiatrist provided input before the meeting via email

MDT involving infectious diseases physician, two respiratory physicians and radiologist

MDT meeting involving nephrologist, hepatologist, haematologist and histopathologist

MDT meeting involving representatives from neurology, immunology, general surgery and clinical pharmacology

MDT meeting involving gastroenterologist, nurse consultant in nutrition, dieticians at tertiary unit, surgeon and psychiatrist at referring hospital, and GP

\section{Outcome}

Consensus diagnosis of somatisation illness made and coordinated follow-up plan agreed

Joint agreement to treat for tuberculosis and coordinated plan for follow-up after 3 months

MDT concluded unlikely underlying microangiopathy; agreed joint plan for further investigation; referral to gastroenterologist to exclude protein-losing enteropathy

Targeted investigations to confirm and refute various differential diagnoses; clarification of prognosis and plans for discharge; discharged 10 days after MDT

Discussion revealed significant premorbid psychological issues; agreed joint plan for investigation and management across sites

Ex-vMDT = extraordinary virtual multidisciplinary team; MDT = multidisciplinary team

able to achieve material improvements in the quality of care (Fig 2), but that these advances would also have been unlikely to occur without the Ex-vMDT. Clinicians felt that such a system was needed and valued the support provided by the MDT coordinator. They were satisfied with the ability to participate from any location and the ease of joining the meeting, but felt the referral process and audiovisual technicalities needed to be improved.

One clinician remarked:

All members of the MDT felt that it was extremely useful and helpful process allowing clear discussions between each specialty team. Most importantly, it allowed us to move to planning discharge and the patient was discharged within ten days to neuro-rehab after having been in hospital for 6 months! Without the MDT this would not have happened.

\section{Patient feedback}

Feedback was obtained from two of the five patients discussed. Both patients indicated that they valued the meeting and felt that it improved their experience of care.

One commented:

Excellent. Care was well coordinated after the MDT. Everybody knew what they were doing. I also knew what was going on as the letter which was sent to me was put in a language that I could understand.

\section{Another remarked:}

When in hospital there were times when different teams had to wait until they could meet with other teams for example infection teams had to wait to speak with liver team and kidney team and it took a longer time. Using the new way, they don't have to be in the same room.

\section{Challenges to implementation}

\section{Establishing the Ex-vMDT system}

The process of setting up the Ex-vMDT system took 6 months and was substantially delayed by the time taken to receive local information governance approval. The use of video-conferencing software from an external provider to discuss confidential information was an understandable concern that had to be fully assessed. National approval and procurement of appropriate software could obviate the need for similarly protracted approval processes in other hospitals.

\section{Coordination of Ex-vMDT}

Identifying and agreeing a suitable time and date for the meetings between several clinicians sometimes working across multiple sites proved a significant logistical challenge; sometimes, even identifying a suitable clinician within each specialty to participate proved difficult. On-call clinicians were often unable to commit to a scheduled meeting because of the unpredictable nature of their workload.

The composition of any MDT is an important consideration: if too small, it can lack the breadth of expertise required to deal with complex problems; if too large, communication can be impaired and reaching a consensus plan can be more difficult. ${ }^{1,10}$ In this pilot, we left it to the discretion of the requesting clinician to decide on the invitees. In two of the five meetings conducted, several key parties failed to attend. We would recommend that referring clinicians restrict participation to essential members to facilitate timely scheduling. As with any MDT meeting, the effectiveness of the Ex-vMDT is dependent on leadership.,10 Although in this pilot we provided the support of a MDT coordinator for logistical and technological issues, 
Fig 2. Residual bar charts showing feedback from nine participants in the extraordinary virtual multidisciplinary team (MDT) meeting pilot in the following domains: (a) technology; (b) process; and (c) outcome. Respondents rated agreement with individual statements using a 5-point scale ('Not at all', 'Somewhat', 'Not applicable', 'Very', and 'Extremely'). Residual values calculated by subtracting 3 (ie mid-point 'Not applicable' score) from the weighted average of responses. Areas with which respondents were very or extremely satisfied are shown in green and not at all or only somewhat satisfied are shown in red. ICE $=$ Hospital electronic system for laboratory, radiology requests and specialty referrals. (a) Technology - How satisfied are you with GoToMeeting with the:

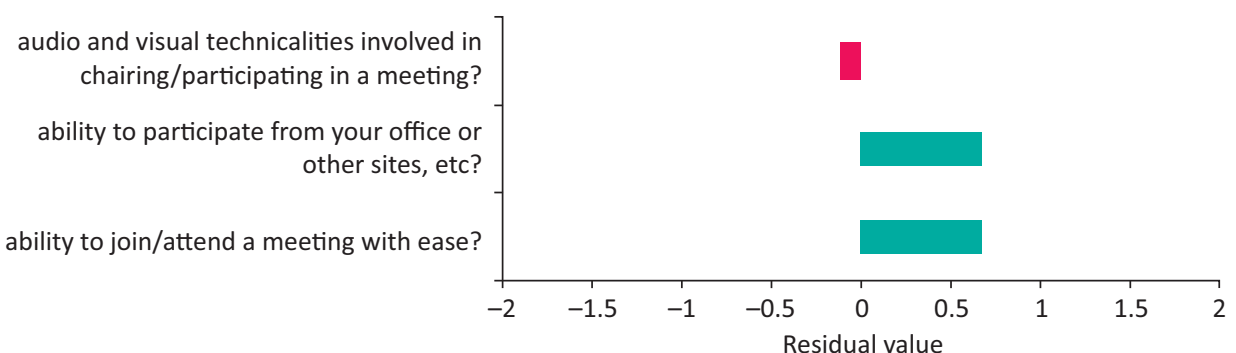

(b) Process - How satisfied are you with:

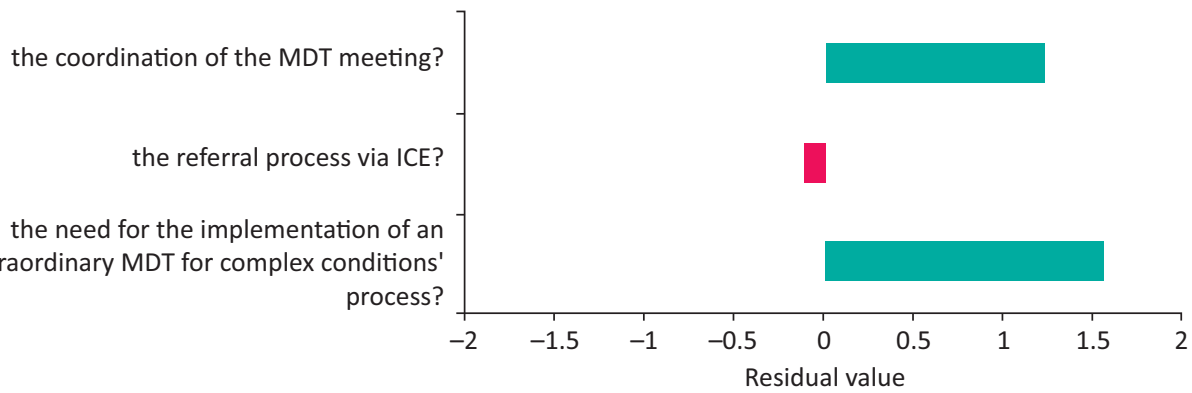

(c) Outcome - How satisfied are you with:

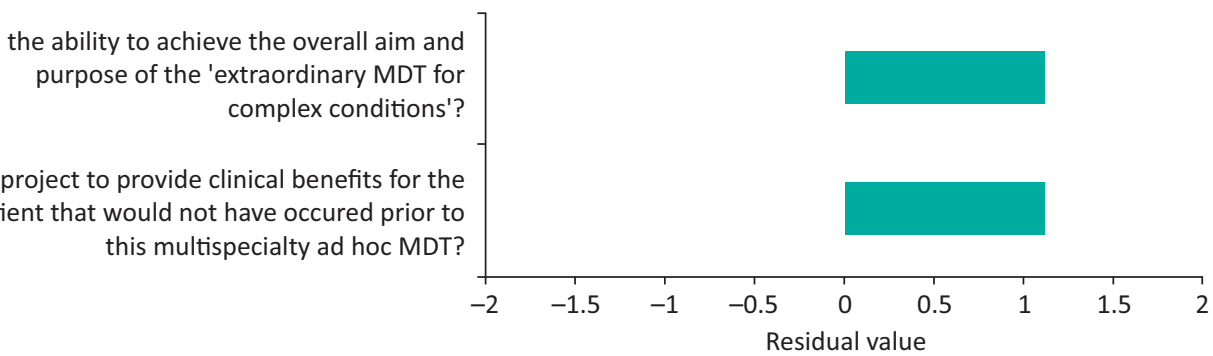

the active engagement of the lead clinician in facilitating, planning and running the Ex-vMDT meeting was vital. Direct communication between the lead clinician and invitees ahead of contact by the MDT coordinator eased the planning of the meeting.

\section{Conduct of the MDT - technology}

In this pilot study, deficiencies in the quality of the audiovisual link were the key criticism of participants and we will address this as a priority in further developing the service. Although virtual meetings have successfully been adopted as a feasible alternative to traditional face-to-face meetings in a variety of settings, 14,15 they present specific challenges to effective communication. Even with modern video-conferencing systems, some of the richness of information conveyed by nonverbal cues is lost and effective communication constrained. ${ }^{10}$ Participants might unconsciously respond to the limitations of system by adopting a more assertive form of conversation that could inhibit discussion. ${ }^{10}$ The impact of the intrinsic limitations of the medium on the effectiveness of the meeting are amplified by any technical difficulties. ${ }^{16}$ These challenges are further accentuated by the context of an extraordinary meeting, in which the participants might not know each other.

\section{Plans for further development}

\section{Overcoming barriers to use}

Although widely regarded as a potentially useful innovation, the Ex-vMDT system was not universally welcomed. The fear of getting inundated with requests to join such meetings was raised by several clinicians. Clinicians who participated in the meetings did so during their free time; there was no mechanism for their departments to be remunerated for their contribution. If Ex-vMDT were to be used widely, the activity would need to be appropriately incentivised. $^{10}$

During the pilot, only physicians requested Ex-vMDT. Further work is needed to understand why surgeons did not use the ExVMDT and whether the innovation could be adapted to suit their needs and those of their patients. It is likely that many clinicians already use informal virtual networks of case discussion (eg via email) to discuss challenging clinical scenarios with colleagues in other specialties. For the Ex-vMDT format to be widely adopted, clinicians will need to be convinced that this forum, which supports synchronous discussion in real-time, provides genuine added value to patient care over these existing methods of communication. 
Lack of familiarity with audiovisual-conferencing technology might have contributed to the reticence to participate expressed by some clinicians. The use of video-conferencing technologies or other forms of virtual working is already widespread in healthcare and will doubtless increase. ${ }^{17}$ Suitable training and support packages are needed to overcome this barrier. Some medical schools have already incorporated practical case-based teaching programs on virtual team working into their undergraduate curricula. $^{18}$

\section{Integration of primary and secondary care}

Although the initial objective of the Ex-vMDT meeting was to improve care coordination between specialties within secondary care, several of the patients discussed had complex needs that required input from primary care and mental health services. Inefficient information flow between care providers in different sectors (eg physical and mental health services) is a key barrier to delivering effective integrated care for patients with complex needs. ${ }^{2,11}$ Similar vMDTs could greatly facilitate coordinated care for selected patients.

\section{Shared decision making}

The lack of direct patient participation is a potential shortcoming of the MDT approach to case management. ${ }^{19}$ In this pilot, we provided patients with written documentation of the Ex-vMDT meeting and invited their feedback. If a vMDT format was widely adopted, mechanisms to promote shared decision making should be developed. ${ }^{20}$

\section{Developing key performance indicators}

There are opportunity costs associated with MDT meetings and, although a virtual format mitigates some of these, a robust series of performance measures are nonetheless required. Process measures might include evidence that: referrals are processed promptly; all relevant parties attend; and the technology functions effectively. Potential outcome measures could include: the meeting is convened within a suitable time frame; evidence that the MDT recommendation was acted upon; and patient satisfaction with the decision-making process. $^{10}$

\section{Conclusion}

The availability of versatile and affordable digital conferencing technologies presents an excellent opportunity to improve interspecialty collaboration and enhance the integration, timeliness and quality of healthcare delivery for patients with complex needs.

\section{Key messages}

> We have developed and successfully piloted the concept of an extraordinary virtual MDT meeting for complex conditions.

> We have shown that such a virtual MDT is valued by clinicians and patients and can improve diagnosis, treatment and discharge planning.

> Further research is needed to assess its utility when used on a large scale and, also explore how it could be used for surgical patients, paediatric patients and within primary and social care.
> Patient involvement and feedback also needs to be developed and assessed further.

\section{Author contributions}

EN, CW and SR developed the original concept of the Ex-vMDT. SJA and $A D$ helped set up and pilot the video-conferencing system. BP is the Ex-vMDT coordinator. EN and SR conducted the quantitative and qualitative analysis of Ex-VMDT. SR and SJA wrote the first draft of this article. All authors have contributed to this paper and concur on its content. EN is guarantor. The views expressed in this publication are those of the authors and not necessarily those of their employers.

\section{Conflicts of interest}

2Bio Limited was contracted to provide innovation project management support by Royal Liverpool and Broadgreen University Hospitals NHS Trust and has no beneficial interests in the outputs of this work.

\section{References}

1 Ruhstaller T, Roe H, Thurlimann B, Nicoll J]. The multidisciplinary meeting: an indispensable aid to communication between different specialities. Eur J Cancer 2006;42:2459-62.

2 Lewis $G$, Vaithianathan R, Wright L et al. Integrating care for highrisk patients in England using the virtual ward model: lessons in the process of care integration from three case sites. Int J Integr Care 2013;13:e046.

3 Ke KM, Blazeby JM, Strong S et al. Are multidisciplinary teams in secondary care cost-effective? A systematic review of the literature. Cost Eff Resource Alloc 2013;11:7.

4 Taylor C, Munro AJ, Glynne-Jones R et al. Multidisciplinary team working in cancer: what is the evidence? BMJ 2010;340:c951.

5 Department of Health. The NHS Cancer Plan: a plan for investment; a plan for reform. London: NHS, 2000.

6 Friedland PL, Bozic B, Dewar ] et al. Impact of multidisciplinary team management in head and neck cancer patients. $\mathrm{Br}$ ] Cancer 2011;104:1246-8.

7 Kesson EM, Allardice GM, George WD, Burns HJ, Morrison DS. Effects of multidisciplinary team working on breast cancer survival: retrospective, comparative, interventional cohort study of 13,722 women. BMJ 2012;344:e2718.

8 Munro A, Brown M, Niblock P, Steele R, Carey F. Do multidisciplinary team (MDT) processes influence survival in patients with colorectal cancer? A population-based experience. BMC Cancer 2015;15:686.

9 Basta YL, Bolle S, Fockens P, Tytgat KM. The value of multidisciplinary team meetings for patients with gastrointestinal malignancies: a systematic review. Ann Surg Oncol 2017;24:266978.

10 Munro AJ, Swartzman S. What is a virtual multidisciplinary team (vMDT)? Br J Cancer 2013;108:2433-41.

11 Goodwin N, Sonola L, Thiel V, Kodner DL. Coordinated care for people with complex chronic conditions. Key lessons and markers for success. London: The King's Fund, 2013.

12 Imison C, Sonola L, Honeyman M, Ross S. The reconfiguration of clinical services. What is the evidence? London: The King's Fund, 2014.

13 NHS England. Five Year Forward View. London: NHS, 2014.

14 Marshall CL, Petersen NJ, Naik AD et al. Implementation of a regional virtual tumor board: a prospective study evaluating feasibility and provider acceptance. Telemed J E Health 2014;20:705-11. 
15 Bolle SR, Larsen F, Hagen O, Gilbert M. Video conferencing versus telephone calls for team work across hospitals: a qualitative study on simulated emergencies. BMC Emerg Med 2009;9:22.

16 Demiris G, Oliver DP, Wittenberg-Lyles E, Washington K. Videomediated communication in hospice interdisciplinary team meetings: examining technical quality and content. AMIA Annu Symp Proc 2009:135-9.

17 Axford AT, Askill C, Jones AJ. Virtual multidisciplinary teams for cancer care. J Telemed Telecare 2002;8(Suppl 2):3-4.

18 Dow AW, Boling PA, Lockeman KS et al. Training and assessing interprofessional virtual teams using a web-based case system. Acad Med 2016;91:120-6.
19 Thornton S. Time to review utility of multidisciplinary team meetings. BMJ 2015;351:h5295.

20 Coulter A, Collins A. Making shared decision-making a reality. No decision about me, without me. London: The King's Fund, 2011.

Address for correspondence: Dr Emmanuel Nsutebu, Tropical and Infectious Diseases Unit, Royal Liverpool and Broadgreen University Hospitals NHS Trust, Prescot Street, Liverpool L7 8XP, UK.

Email: Emmanuel.Nsutebu@rlbuht.nhs.uk

\title{
Royal College of Physicians
}

\section{Physicians and war}

\author{
War has been a frequent feature of British life over \\ the 500 years of the Royal College of Physicians' \\ existence, and has an important place in the histories \\ of both the RCP and medicine.
}

Order by phone or online

Tel +44 (0)20 30751358

or visit www.rcplondon.ac.uk/shop

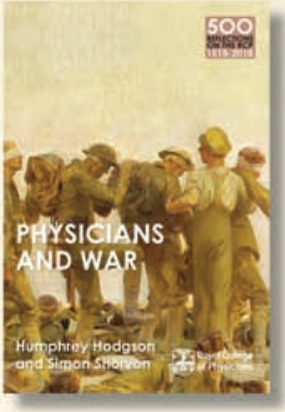

Price: $€ 12$

(Postage and packaging not included)

$10 \%$ discount for fellows and members.

$15 \%$ discount for foundation doctors and students 